\title{
Risk Factors for Surgical Site Infection After Appendectomy for Acute Appendicitis; Results of a Cross-Sectional Study Carried out at a Community Hospital in Qatar (2013-2016)
}

\author{
Humberto Guanche Garcell ${ }^{1 *}$, Ariadna Villanueva Arias ${ }^{1}$, Cristobal A. Pancorbo Sandoval ${ }^{2}$, Adan Bode

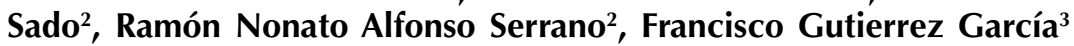

'Infection Control Department, The Cuban Hospital, Hamad Medical Corporation, Doha, Qatar ${ }^{2}$ Surgical Department, The Cuban Hospital, Hamad Medical Corporation, Doha, Qatar

${ }^{3}$ Hospital Epidemiology Department, Hospital Joaquín Albarrán, La Habana, Cuba

*Corresponding Author: Humberto Guanche Garcell, M.D., Professor, Infection Control Department, The Cuban Hospital, Hamad Medical Corporation, Doha, Qatar. Tel: +535-5013515, Email: humbertoguanchegarcell@yahoo.es, guanche@infomed.sld.cu

Received August 28, 2018; Accepted February 23, 2019; Online Published April 15, 2019

\begin{abstract}
Background: Identifying risk factors for surgical site infection (SSI) after appendectomies could be useful in focusing prevention programs.

Objective: The current study aimed to identify the risk factors associated with SSI in a cohort of patients from a single center in Western Qatar.

Methods: This cross-sectional study was carried out at The Cuban Hospital (Dukhan, Qatar) on patients who had undergone an appendectomy from January 2013 through September 2016. Data extracted from the patient files included demographics, comorbidities and toxic habits, surgery type, procedure duration, wound type, appendicitis-type, American Anesthesiology Score, body mass index, selected laboratory tests, and compliance with antibiotic prophylaxis.

Results: The study variables, type of surgery performed, procedure duration, wound and appendicitis type, serum albumin, and timing of antibiotic prophylaxis, were found to be related to the occurrence of SSI in univariate analysis $(P=0.000)$. Logistic regression showed that open appendectomies (odds ratio $[\mathrm{OR}]=22.90,95 \% \mathrm{Cl} ; 8.04-65.21$ ), low serum albumin $(\mathrm{OR}=0.92,95 \% \mathrm{Cl}$; 0.85-0.99), and improper timing of antibiotic prophylaxis $(\mathrm{OR}=44.92,95 \% \mathrm{Cl}$; 3.39-594.91) were independently associated with the occurrence of SSI.

Conclusion: Cases of complex appendicitis, open procedure, low serum albumin level, and improper timing of antibiotic prophylaxis constituted the risk factors for SSI in the setting of this study. The infection control program should focus on improving the quality of antibiotic prophylaxis and closely monitoring patients who undergo open surgical procedures.

Keywords: Appendicitis, Appendectomy, Surgical Site Infection, Risk Factors, Qatar
\end{abstract}

\section{Background}

Acute appendicitis is the most common cause of emergency surgical procedures in general hospitals, and most procedures are performed laparoscopically. The incidence of surgical site infection in appendectomies is variable in different settings. Lower infection rates have been reported by the National Healthcare Safety Network (Center for Disease Control and Prevention/United States) and the International Nosocomial Infection Control Consortium (1.4\% and $2.9 \%$, respectively). ${ }^{1}$

The risk of acquiring a surgical site infection (SSI) depends on patient-related factors (e.g., age, the presence of comorbidities) and procedure factors (e.g., procedure duration, wound type, compliance with antibiotic prophylaxis). ${ }^{2}$ In appendectomies, others factors have also been reported, including pre-existing diabetes mellitus, ${ }^{3-5}$ obesity, and nutritional factors. ${ }^{5}$ Complicated appendicitis, especially gangrenous and perforated types, is related to greater risk of SSI regardless of the operative procedure. ${ }^{1,6}$

In appendectomies, laparoscopy provides several advantages, among which a reduced SSI rate is the most valuable. ${ }^{7,8}$ Nevertheless, even though a wide spectrum of appendicitis is managed with this technique, the risk of postoperative intra-abdominal abscess and SSI in complex

Copyright (C 2019 The Author(s). This is an open-access article distributed under the terms of the Creative Commons Attribution License (http:// creativecommons.org/licenses/by/4.0), which permits unrestricted use, distribution, and reproduction in any medium, provided the original work is properly cited. 
appendicitis should be considered. ${ }^{8,9}$

\section{Objective}

This prospective study was conducted on a cohort of consecutive patients who underwent appendectomy for acute appendicitis in a single center in Western Qatar with the objective of identifying the risk factors associated with SSI.

\section{Methods}

This cross-sectional study was carried out at The Cuban Hospital (TCH) (Dukhan, Qatar) on patients who had undergone an appendectomy from January 2013 through September 2016. TCH is a 75-bed community hospital that provides healthcare services for patients from neighboring communities in Western Qatar and transfers from other facilities.

All patients who had been diagnosed with acute appendicitis and undergone an appendectomy were included in the study. Diagnoses of appendicitis were confirmed by considering the clinical picture, laboratory and radiological findings suggestive of acute inflammation of the appendix.

Demographic data (age, gender), history of active smoking or diabetes mellitus at admission, surgery type (open or laparoscopic appendectomies), procedure duration, wound type (clean-contaminated, contaminated, dirty), appendicitis-type according to surgical findings (flemonous, suppurative, gangrenous, perforated), and American Anesthesiology Score were extracted from patient files. The body mass index was considered to be low at $<19 \mathrm{~kg} / \mathrm{m}^{2}$, normal at $19-30 \mathrm{~kg} / \mathrm{m}^{2}$, and high at $>30 \mathrm{~kg} /$ $\mathrm{m}^{2}$. The results of selected laboratory tests (hemoglobin, creatinine, the absolute count of lymphocytes, serum protein total, serum albumin) performed during the preoperative evaluation were collected. Compliance with antibiotic prophylaxis was monitored by an infectioncontrol practitioner on an ongoing basis and according to the corporate policy recommending a single dose of cefuroxime plus metronidazole within 1 hour before the surgical incision for all appendectomies or the maximum of up to 24 hours, if required. In appendectomies for gangrenous and perforated appendicitis, a full course of antimicrobial treatment is recommended. Compliance with antibiotic prophylaxis was calculated for the timing of administration, selection, dose, and discontinuation; in all cases, the number of compliant prescriptions was divided by the number of procedures (per 100 procedures). The procedures for surveillance of SSIs have previously published. ${ }^{1}$

All statistical analyses were performed using SPSS $\mathbb{R}$ version 22.0 (IBM, Armonk, NY, USA). Continuous data was presented as mean and standard deviation and analyzed using the non-parametric Wilcoxon-ManWhitney test. A homogeneity test was performed on categorical data. After univariate analysis, type of surgery, duration of surgical procedure, wound type, type of appendicitis, serum albumin, and timing of antibiotic prophylaxis were selected for multivariable analysis and entered into a logistic regression model. Prior to analysis, the existence of collinearity between these independent variables was tested. After that, using stepwise backward elimination, the best model was selected. A factor was considered independently associated with the occurrence of SSI if the 95\% CI for the calculated odds ratio (OR) did not include the unity. For all hypothesis tests performed, a significance level of $P=0.05$ was set.

\section{Results}

SSI was reported in 29 patients (2.82\%). Table 1 shows the univariate analysis of the possible factors associated with SSI in appendectomies. As can be observed, the type of surgery performed, procedure duration, wound and appendicitis type, serum albumin, and timing of antibiotic prophylaxis were variables found to be related to the occurrence of this complication $(P=0.000)$. It can be noted that the frequency of SSI is much greater when the type of surgery performed is open $(20.8 \%)$ compared with laparoscopic surgery (1.5\%). In patients who had SSI, the mean procedure duration was 72.7 minutes, in contrast with patients who did not, for whom the procedure duration was lower (39.5 minutes). The lower frequency of SSI was also observed in patients with clean-contaminated wound (1.2\%), and the highest frequency was seen in patients with dirty wound (8.8\%). Contaminated wound was found to have an intermediate frequency (4.5\%). Cases of non-complicated appendicitis and cases of flemonous and suppurative appendicitis had relatively low SSI rates $(0.9 \%$ and $1.7 \%$, respectively), while the frequency of infection was $4.7 \%$ in cases of gangrenous appendicitis and $11.5 \%$ in cases of perforated appendicitis. Mean serum albumin levels were higher in patients without infection $(40.9 \mathrm{~g} / \mathrm{L})$ when compared with patients who had the complication $(35.7 \mathrm{~g} / \mathrm{L})$. Also, the improper timing of antibiotic prophylaxis was related with a higher infection rate $(23.1 \%)$ compared with those with correct timing $(2.6 \%)$. For the other variables, no association with the occurrence of SSI was found.

Multivariate analysis (Table 2) found that the variables type of surgery, serum albumin, and timing were independently associated with the occurrence of SSI. The probability of occurrence of SSI is almost 23 times greater when the type of surgery is open $(\mathrm{OR}=22.90,95 \% \mathrm{CI}$, 8.04-65.21) than when it is laparoscopic. As for serum albumin, it can be seen that for each gram of increase, the risk of SSI decreases by $8 \%(\mathrm{OR}=0.92,95 \% \mathrm{CI}, 0.85-0.99)$, and with improper timing of prophylactic antibiotics, the probability of occurrence of SSI is 44.92 times greater than when it is correct $(\mathrm{OR}=44.92,95 \% \mathrm{CI}, 3.39-594.91)$.

\section{Discussion}

The current study showed that SSI occurs in $2.8 \%$ of patients who undergo appendectomy, and the incidence of SSI is related mainly to non-modifiable risk factors, including open procedures, complex appendicitis, and 
Table 1. Univariate Analysis of Possible Factors Associated With Surgical Site Infection in Appendectomies

\begin{tabular}{|c|c|c|c|}
\hline \multirow{2}{*}{ Variable } & \multicolumn{2}{|c|}{ Surgical Site Infections } & \multirow{2}{*}{$P$ Value } \\
\hline & Yes & No & \\
\hline Age $(y)$ & $31.8(8.9)^{*}$ & $30.6(8.1)^{*}$ & 0.506 \\
\hline \multicolumn{4}{|l|}{ Gender } \\
\hline Male & $29(3.0)$ & $950(97.0)$ & 0.435 \\
\hline Female & 0 & $49(100)$ & \\
\hline \multicolumn{4}{|l|}{ Smoking } \\
\hline Yes & $9(4.4)$ & $195(95.6)$ & 0.125 \\
\hline No & $20(2.4)$ & $804(97.6)$ & \\
\hline \multicolumn{4}{|l|}{ Diabetes mellitus } \\
\hline Yes & $2(6.9)$ & $27(93.1)$ & 0.179 \\
\hline No & $27(2.7)$ & $972(97.3)$ & \\
\hline \multicolumn{4}{|l|}{ Surgery type } \\
\hline Open & $15(20.8)$ & $97(79.2)$ & 0.000 \\
\hline Laparoscopic & $14(1.5)$ & $942(98.5)$ & \\
\hline Procedure duration (min) & $72.7(35.0)^{*}$ & $39.5(22.1)^{*}$ & 0.000 \\
\hline \multicolumn{4}{|l|}{ Wound type } \\
\hline Clean contaminated & $9(1.2)$ & $727(98.8)$ & 0.000 \\
\hline Contaminated & $6(4.5)$ & $126(95.5)$ & \\
\hline Dirty & $14(8.8)$ & $146(91.3)$ & \\
\hline \multicolumn{4}{|l|}{ Appendicitis type } \\
\hline Flemonous & $4(0.9)$ & $436(99.1)$ & 0.000 \\
\hline Suppurative & $6(1.7)$ & $341(98.3)$ & \\
\hline Gangrenous & $6(4.7)$ & $121(95.3)$ & \\
\hline Perforated & $13(11.5)$ & $100(88.5)$ & \\
\hline \multicolumn{4}{|l|}{ ASA } \\
\hline 1 & $17(2.8)$ & $592(97.2)$ & 0.743 \\
\hline 2 & $11(2.7)$ & $397(97.3)$ & \\
\hline 3 & $1(10.0)$ & $9(90.0)$ & \\
\hline 4 & 0 & $1(100)$ & \\
\hline \multicolumn{4}{|l|}{ Laboratory tests } \\
\hline Hemoglobin (g/L) & $15.0(1.2)^{*}$ & $15.0(1.3)^{*}$ & 0.440 \\
\hline Creatinine, serum (mmol/l) & $85.2(3.2)^{*}$ & $82.7(17.2)^{*}$ & 0.847 \\
\hline Lymphocytes, absolute count & $1575(141)^{*}$ & $1570(23)^{*}$ & 0.237 \\
\hline Protein total, serum $(\mathrm{g} / \mathrm{L})$ & $60.4(10.0)^{*}$ & $72.4(7.6)^{*}$ & 0.683 \\
\hline Albumin serum $(g / L)$ & $35.7(7.8)^{*}$ & $40.9(5.2)^{*}$ & 1.000 \\
\hline Body mass index & $26.0(4.5)$ & $24.2(4.3)$ & 0.439 \\
\hline Low & $1(1.2)$ & $84(98.8)$ & \\
\hline Normal & $24(2.9)$ & $818(97.1)$ & \\
\hline High & $4(4.4)$ & $87(95.6)$ & \\
\hline \multicolumn{4}{|c|}{ Compliance with prophylactic antibiotics } \\
\hline \multicolumn{4}{|l|}{ Timing } \\
\hline Correct & $26(2.6)$ & $989(97.4)$ & 0.000 \\
\hline Incorrect & $3(23.1)$ & $10(76.9)$ & \\
\hline \multicolumn{4}{|l|}{ Selection and dose } \\
\hline Correct & $29(2.8)$ & $997(97.2)$ & 0.809 \\
\hline Incorrect & $0(0.0)$ & $2(100)$ & \\
\hline \multicolumn{4}{|l|}{ Discontinuation } \\
\hline Correct & $27(2.7)$ & $962(97.3)$ & 0.375 \\
\hline Incorrect & $2(5.1)$ & $37(94.9)$ & \\
\hline
\end{tabular}

Data is presented as No. (\%) unless specified. *Mean (standard deviation).
Table 2. Odds Ratio, Cl 95\%, of Surgical Site Infection in Appendectomies, Estimated by Logistic Regression

\begin{tabular}{lcc}
\hline Variable & OR & $\mathbf{9 5} \% \mathbf{C l}$ \\
\hline Surgery type & 22.90 & $8.04-65.21$ \\
Open & 1 & --- \\
$\quad$ Laparoscopic* & 1.00 & $0.98-1.02$ \\
Procedure duration (min) & & \\
Wound type & 0.21 & $0.02-2.38$ \\
Contaminated & 0.47 & $0.09-2.50$ \\
Dirty & 1 & --- \\
$\quad$ Clean contaminated* & & $0.46-9.57$ \\
Appendicitis type & 2.1 & $0.58-6.78$ \\
Gangrenous & 1.97 & --- \\
Perforated & 1 & $0.85-0.99$ \\
Flemonous-suppurative* & 0.92 & -- \\
\hline Albumin serum (g/L) & & $3.39-594.91$ \\
Timing & 44.92 & \\
Incorrect & 1 & \\
Correct* & & \\
\hline
\end{tabular}

low serum albumin. The improper timing of prophylactic antibiotics was the modifiable risk factor identified in relation with SSI.

The incidence of SSI in the studied cases was similar to previous reports. ${ }^{10,11}$ This finding is related to the performance of the local infection control program that included surveillance of SSIs and monitoring of compliance with surgical bundles (antibiotic prophylaxis, perioperative glucose control, postoperative normothermia, and hair removal practices).

The literature on appendectomies is consistent regarding the higher incidence of SSI in complex appendicitis and open procedures, while laparoscopic procedures are related to a reduction of this adverse outcome. ${ }^{7,8}$ An important finding to consider is the frequency of complex appendicitis in the setting of the current study ( $40 \%$ of cases). Studies have described lower frequencies of complicated appendicitis, ranging from $11.8 \%$ to $26.5 \%$ of patients. ${ }^{79,12}$ The frequency of complicated appendicitis constitutes an important factor related to the risk of infection but primarily is not a modifiable risk factor. Nevertheless, the possibilities of the best strategies and procedures for local contamination control could contribute to the prevention of infection. Although the frequency of complicated appendicitis is not a primary objective of the current study, the findings could be related to patient factors related to healthcare seeking behavior that will require further study.

Nutritional factors have been related to the incidence of SSI in many surgical procedures, among which are underweight, obesity, and low serum albumin..$^{8,13-15}$ The majority of the patients studied had a normal body mass index with a relatively low frequency of obesity (8.9\%). The diversity of the nutritional habits and the socioeconomic characteristics (mainly expatriate population with low 


\section{Research Highlights}

\section{What Is Already Known?}

Appendectomy is the most common emergency operation performed worldwide. Surgical site infections considered as a frequent adverse event of surgical procedures and incidence of surgical site infection is related with risk factors including patient and procedure factors.

\section{What This Study Adds?}

The risk of surgical site infection is related with complex appendicitis, open procedures, low serum albumin and inadequate timing of prophylactic antibiotics in the study population. Quality improvement process are required to increase antibiotic prophylaxis compliance.

income) of the population could explain this finding.

In general, the antibiotic prophylaxis constituted a preventive practice for SSI with special reference to the timing of administration. ${ }^{16}$ Sánchez-Santana et al, BerríosTorres et al, and $\mathrm{Wu}$ et al did not demonstrate a relation between the timing of antibiotic and the incidence of SSI. Nevertheless, the proper timing of administration in order to achieve the bactericidal concentration of the prophylactic agents in the serum and tissues when the incision is made is a well-accepted practice. ${ }^{16-18}$ The prolongation of antibiotic treatment more than 24 hours post-procedure is indicated in cases of contaminated and dirty appendicitis, but a few studies did not show its preventive effect for an intra-abdominal abscess. ${ }^{12}$

Important limitations of this study should be emphasized. First, the study variables include well-known risk factors for SSIs, but other patient or procedure factors could be considered. Population behaviors could be important to consider in addition to nutritional markers and the quality of postoperative wound care. Second, this was a singlecenter study, which limits data comparison, especially when taking into account the patients' profiles.

\section{Conclusion}

The results of the current study show that complex appendicitis, open procedures, low serum albumin, and improper timing of antibiotic prophylaxis constituted the risk factors for SSI in the setting of the study. The infection control program should focus on improving the quality of antibiotic prophylaxis and the close monitoring of patients who undergo open surgical procedures.

\section{Authors' Contributions}

HGG: research design, data collection, analysis, and writing; AVA: research design, data collection, analysis, review of the manuscript; CAPS: data collection, analysis, review of the manuscript; ABS: data collection, analysis, review of the manuscript; RNAS and FGG: research design, data collection, analysis, and writing.

\section{Conflict of Interest Disclosures}

The authors declare that they have no conflicts of interest.

\section{Ethical Approval}

The study received ethical approval from the Quality Management Department of $\mathrm{TCH}$.

\section{Acknowledgments}

The authors would like to thank Mr. Carlos L. Crespo Palacios for reviewing this paper.

\section{References}

1. Garcell HG, Arias AV, Sandoval CA, et al. Incidence and Etiology of Surgical Site Infections in Appendectomies: A 3-Year Prospective Study. Oman Med J. 2017;32(1):31-35. doi:10.5001/omj.2017.06.

2. Mangram AJ, Horan TC, Pearson ML, Silver LC, Jarvis WR. Guideline for Prevention of Surgical Site Infection, 1999. Centers for Disease Control and Prevention (CDC) Hospital Infection Control Practices Advisory Committee. Am J Infect Control. 1999;27(2):97-132; quiz 133-134; discussion 196. doi:10.1016/S0196-6553(99)70088-X.

3. Ming PC, Yan TY, Tat LH. Risk factors of postoperative infections in adults with complicated appendicitis. Surg LaparosC Endosc Percutan Tech. 2009;19(3):244-248. doi:10.1097/ SLE.0b013e3181a4cda2.

4. Martin ET, Kaye KS, Knott C, et al. Diabetes and Risk of Surgical Site Infection: A Systematic Review and Meta-analysis. Infect Control Hosp Epidemiol. 2016;37(1):88-99. doi:10.1017/ ice.2015.249

5. Cho M, Kang J, Kim IK, Lee KY, Sohn SK. Underweight body mass index as a predictive factor for surgical site infections after laparoscopic appendectomy. Yonsei Med J. 2014;55(6):16111616. doi:10.3349/ymj.2014.55.6.1611

6. Giesen LJ, van den Boom AL, van Rossem CC, den Hoed PT, Wijnhoven BP. Retrospective Multicenter Study on Risk Factors for Surgical Site Infections after Appendectomy for Acute Appendicitis. Dig Surg. 2017;34(2):103-107. doi:10.1159/000447647

7. Xiao Y, Shi G, Zhang J, et al. Surgical site infection after laparoscopic and open appendectomy: a multicenter large consecutive cohort study. Surg Endosc. 2015;29(6):1384-1393. doi:10.1007/s00464-014-3809-y.

8. Biondi A, Di Stefano C, Ferrara F, Bellia A, Vacante M, Piazza L. Laparoscopic versus open appendectomy: a retrospective cohort study assessing outcomes and cost-effectiveness. World J Emerg Surg. 2016;11(1):44. doi:10.1186/s13017-016-0102-5.

9. van Rossem CC, Bolmers MD, Schreinemacher $\mathrm{MH}$, van Geloven AA, Bemelman WA. Prospective nationwide outcome audit of surgery for suspected acute appendicitis. Br J Surg. 2016;103(1):144-151. doi:10.1002/bjs.9964.

10. Edwards JR, Peterson KD, MuY, et al. National Healthcare Safety Network (NHSN) report: data summary for 2006 through 2008, issued December 2009. Am J Infect Control. 2009;37(10):783805.doi:10.1016/j.ajic.2009.10.001.

11. Rosenthal VD, Richtmann R, Singh $S$, et al. Surgical site infections, International Nosocomial Infection Control Consortium (INICC) report, data summary of 30 countries, 2005-2010. Infect Control Hosp Epidemiol. 2013;34(6):597604. doi:10.1086/670626.

12. Cho J, Park I, Lee D, Sung K, Baek J, Lee J. Antimicrobial treatment after laparoscopic appendectomy for preventing a post-operative intraabdominal abscess: a prospective cohort study of 1817 patients. Int J Surg. 2016;27:142-146. 
doi:10.1016/j.ijsu.2016.01.069.

13. Brenner P, Nercelles P. Prevention of Surgical Site Infection. In: IFIC Basic Concepts of Infection Control. 2nd ed. International Federation of Infection Control; 2011.

14. Lubega A, Joel B, Justina Lucy N. Incidence and Etiology of Surgical Site Infections among Emergency Postoperative Patients in Mbarara Regional Referral Hospital, South Western Uganda. Surg Res Pract. 2017;2017:6365172. doi:10.1155/2017/6365172.

15. Yuwen $\mathrm{P}, \mathrm{Chen} \mathrm{W}, \mathrm{Lv} \mathrm{H}$, et al. Albumin and surgical site infection risk in orthopaedics: a meta-analysis. BMC Surg. 2017:17(1):7. doi:10.1186/s12893-016-0186-6.
16. Berríos-Torres SI, Umscheid CA, Bratzler DW, et al. Centers for Disease Control and Prevention Guideline for the Prevention of Surgical Site Infection, 2017. JAMA Surg. 2017;152(8):784791. doi:10.1001/jamasurg.2017.0904.

17. Wu WT, Tai FC, Wang PC, Tsai ML. Surgical site infection and timing of prophylactic antibiotics for appendectomy. Surg Infect (Larchmt). 2014;15(6):781-785. doi:10.1089/sur.2013.167.

18. Sánchez-SantanaT, Del-Moral-Luque JA, Gil-Yonte P, BanuelosAndrio L, Duran-Poveda M, Rodriguez-Caravaca G. [Effect of compliance with an antibiotic prophylaxis protocol in surgical site infections in appendectomies. Prospective cohort study]. Cir Cir. 2017;85(3):208-213. doi:10.1016/j.circir.2016.09.004. 